\title{
Earliest Arrival of Evacuees with Contraflow Approach
}

\author{
Shiva Prakash Gupta ${ }^{1}$, Shree Ram Khadka ${ }^{2}$ \\ ${ }^{1}$ Trichandra Multiple Campus, Tribhuvan University, Kathmandu, Nepal \\ ${ }^{2}$ Central Department of Mathematics, Tribhuvan University, P.O. Box 13143, Kathmandu, Nepal \\ Corresponding author: spguptalk@yahoo.com; shreeramkhdka@gmail.com
}

Received: March 4, 2016 Revised: July 19, $2016 \quad$ Accepted: Aug. 5, 2016

\begin{abstract}
The earliest arrival flow problem sends a maximum number of evacuees as early as possible during the evacuation of people and/or property from dangerous zone to the safe zone. The contraflow approach significantly increases the capacity to send the evacuees at one hand and reduces the time the evacuation takes on the other hand. The problem has been extensively studied as an optimization problem with wide application. In this paper we briefly revisit the existing literature of the problem.
\end{abstract}

Keywords: Earliest arrival flow, Contraflow approach, Evacuation problem

\section{Introduction}

People may be in dangerous situation because of different natural and manmade disasters. For example, the recent Nepal earthquake and Paris attack. An efficient evacuation strategy is required to save the lives and property in such a situation. The strategy incorporates the four steps preparedness, planning, response and recovery (PPRR). The preparedness deals with the reduction or elimination of the effects of a hazard. The planning step draws a layout for the efficient evacuation. The response phase involves actions whereas the recovery seeks to bring back the situation into normalcy.

We concentrate on the contraflow approach of the earliest arrival flow problem. This is a part of planning. The problem sends a maximum number of evacuees as early as possible from the disastrous zone to the safe zone. The contraflow approach has a significant impact to increase the capacity of the path as well as to reduce the time to send. Reduction of time is really important to save the lives and/or property of the people in that situation. The problem has been extensively studied even to achieve the optimality. In this paper, we briefly revisit the existing literature with optimization version.

The paper is organized as follows: We formulate earliest arrival flow problems in section 2, solution procedures algorithms and examples in section 3, Contraflow approach and earliest arrival contraflow problems in section 4 . The final section concludes the paper. 


\section{Mathematical Formulation of Earliest Arrival Flow}

The problem has been studied as a network flow in which the disastrous zone $s$, which may be a building or a region or a vehicle, is considered as a source, all the paths the evacuees send through are the $\operatorname{arcs}(x, y)$ with $E=\{(x, y): x, y \in V\}$, where $V$ is the set of nodes $x$ which are the crossings and the safe zone is taken as a sink $t$. We consider the network $N(V, E, c, \tau, s, t$, $T$ ) where $\mathrm{c}$ represents the capacity and $\tau$ represents the transit time of the arc. So in the evacuation problem this method is very useful and be applied so that for each discrete time step maximal number of evacuees can be evacuated from dangerous zone to the safety zone. Linear Programming Formulation of earliest arrival flow problem by David Gale [10] is

maximize $\quad v(\theta)$ for each $\theta, \theta=0,1,2, \ldots, \mathrm{T}$.

such that

$$
\begin{aligned}
& \sum_{\theta=0}^{T} \sum_{y \in V}[f(x, y, \theta)-f(y, x, \theta-\tau(y, x)]=v(\theta) \text { for } x=s \\
& \sum_{y \in V}[f(x, y, \theta)-f(y, x, \theta)-\tau(y, x)]=0 \text { for } x \neq s, t ; \theta=0,1,2, \ldots T \\
& \sum_{\theta=0}^{T} \sum_{y \in V}[f(x, y, \theta)-f(y, x, \theta-\tau(y, x)]=-v(\theta) \text { for } x=s \\
& 0 \leq f(x, y, \theta) \leq c(x, y)
\end{aligned}
$$

For all $\theta \in\{0,1,2, \ldots, T\}$ and $(x, y) \in E$, we have $\tau(x, x)=1, c(x, x)=\infty$ for hold over at node $x$. Equation (1) represents total outflow from source node, equation (2) represents net flow at intermediate nodes, equation (3) represents total inflow towards sink and equation (4) represents capacity constraint. The objective is to maximize the flow as early as possible.

\section{Solution Strategies of the Problem}

The network $N$ on which the problem is described may contain all or some two way arcs. The capacity of the arc can be increased if the arcs are reversed. It has been investigated that the capacity can be doubled and the evacuation time can be halved [13]. The problem has been formulated as a linear programming with efficient algorithm to find the optimal solution $[10,14,21]$. The solution is basically based on the Ford and Fulkerson's algorithm on the dynamic network to find a maximum solution $[8,9]$. Furthermore, an algorithm for the earliest arrival and the latest departure of the flow also exists [12]. The dynamic network $N$ is converted into $\widetilde{N}$ by reversing the arcs.

\section{Algorithm 1: [12] Earliest arrival and latest departure flow algorithm}

Input: Modified time-expanded graph $\tilde{G}(\mathrm{~T})$ 
Output: Earliest arrival and latest departure maximal dynamic flow.

Begin: Apply the maximal dynamic flow algorithm to find a maximal dynamic flow on the underlying static network $\mathrm{N}(\mathrm{V}, \mathrm{E}, \mathrm{s}, \mathrm{t})$

Whenever a flow augmenting path is found, consider the corresponding arcs of each copy of this path in $\tilde{G}(\mathrm{~T})$.

If the arc is forward arc then label it with the time when the flow enters this arc.

Else

Remove the former label

End if

End:

At termination, these labels indicate a latest departure-earliest arrival schedule. The algorithm is pseudo-polynomial that depends on $T$ as in [14,21]. In 1994 Hoppe and Tardos [11] presented the first polynomial time approximation algorithm for the earliest arrival flow problem, which gives a $(1+\epsilon)$ approximation for any fixed $\in>0$. They developed a capacity scaling shortest augmenting path algorithm with the unusual feature of scaling upwards. This means that the scaling start with $\Delta=1$ and increases $\Delta$ by setting $\Delta=2 \Delta$ after iteration until no s - t path of length less or equal $\mathrm{T}$ exists. The algorithm works on the residual network $\widetilde{G}^{\prime}(x)=\left(\mathrm{V}, E^{\prime}(\mathrm{x}), \mathrm{T}\right)$ of the dynamic network $N(V, E, c, \tau, s, t, T)$. Where the capacities arcs updated according to the flow changes and are rounded at the end of each iteration by the scaling increasing scaling factor $\Delta$.

\section{Algorithm 2: [11] Polynomial time approximation algorithm for the earliest arrival flow problem.}

Input: Dynamic network $N(V, E, c, \tau, s, t, T)$ with capacity function $\mathrm{c}$ and transit time function $\tau \forall(\mathrm{i}, \mathrm{j}) \in \mathrm{E}$. chain decomposition set $\mathrm{P}=\varnothing$ and scaling factor $\Delta=1$, rounded capacity $\tilde{c}=\mathrm{c}$ flow $\mathrm{x}=0$ for some $\in>0$.

Output: $(1+\epsilon)$ approximation of the earliest arrival flow.

While there exists a s - t path in $G_{c}(x)$ with length $\leq \mathrm{T}$.

Set $\sigma=0$

\section{While}

$\left(\sigma<\frac{m \Delta}{\epsilon}\right)$ and there exists a s - t path in $\dot{G}_{C}(\mathrm{x})$ with length $\leq T$.

Do

Find the shortest $\mathrm{s}-\mathrm{t}$ path in $\hat{G}_{C}(\mathrm{x})$ and denote it by $\mathrm{p}$.

$l=\left(\tilde{c}_{i j}:(\mathrm{i}, \mathrm{j}) \in \mathrm{P}\right)$.

Augment the flow $\mathrm{x}$ by $l$ along $\mathrm{P}$ and update the chain decomposition

Set $\mathrm{P}=\mathrm{P} \cup\{(l ; p)\}$

$\sigma=\sigma+1$ 
End while

Increase the scaling factor $\Delta=2 \Delta$

Round the residual capacity $\forall(\mathrm{i}, \mathrm{j}) \in E(\mathrm{x})$

$\tilde{c}_{i j}=c_{i j}-\left(c_{i j} \bmod \Delta\right)$

End while

If $\mathrm{P} \neq \varnothing$ the dynamic flow $\mathrm{f}$ can be obtained by the repeating all path flows in $\mathrm{P}$.

Example 1: Let us consider the graph shown in Figure 3 with time horizon $\mathrm{T}=7$ and $\in=1.25$ to see how above algorithm works. First we set the scaling factor $\Delta=1$, the flow $\mathrm{f}(\mathrm{x}, \mathrm{y})=0$ and $\mathrm{P}$ $=\varnothing$, we start with $\sigma=0$ and we have $\frac{m \Delta}{\epsilon}=\frac{5 \times 1}{1.25}=4$. Here $\left(\sigma=0<4=\frac{m \Delta}{\epsilon}\right)$.

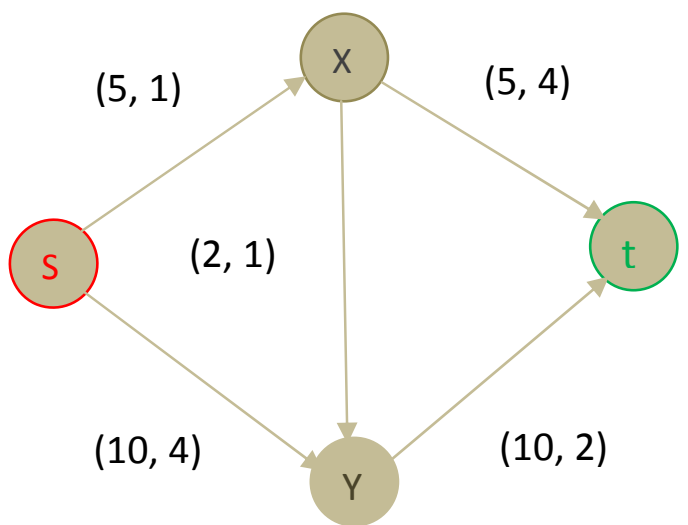

Fig. 3: (a) Dynamic Network

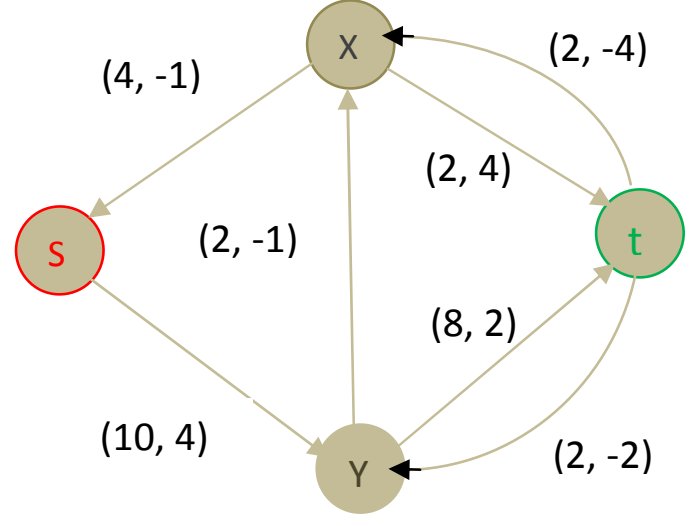

(b) Network after First Rounding

So we apply the inner loop.

The shortest path is $P_{1}=\{\mathrm{s}, \mathrm{x}, \mathrm{y}, \mathrm{t}\}$ with length 4 and maximal residual capacity $l=2$, thus we augmenting the flow along $p_{1}$ by $l$, we get the flow value $v(\mathrm{f})=2$ and update the residual capacities. Add $p_{1}$ to the chain decomposition set $\mathrm{P}$ and $\sigma=\sigma+2$. Since also $\sigma=2<4=\frac{m \Delta}{\epsilon}$ and we have $\mathrm{s}-\mathrm{t}$ path $P_{2}=\{\mathrm{s}, \mathrm{x}, \mathrm{t}\}$ with length 5 and flow value 3 . Update the residue network and set $\sigma=\sigma+3=5$. Here $\sigma<\frac{m \Delta}{\epsilon}$ is not possible. So we have to increase the scaling factor set $\Delta=2 \Delta$ and round the capacities as shown in figure. In the next iteration there exists a s - t path $P_{3}=\{\mathrm{s}, \mathrm{y}, \mathrm{t}\}$ with length $\mathrm{G}$ and flow value 8 in the rounded residual network. We set $\sigma=0$ and we find $\frac{m \Delta}{\epsilon}=\frac{2 \times 5}{1.25}=8$, here $\sigma<\frac{m \Delta}{\epsilon}$. So we update the flow value $\mathrm{V}$ (f) $=5+8=13$ and the residual capacities. Then we set: $\sigma+8=8$ and thus we cannot repeat the inner loop again, since $\sigma<8$ is not possible. We increase the scaling factor and get $\Delta=4$ and round the capacities then we get there does not exists $\mathrm{s}-\mathrm{t}$ path. 


\section{Contraflow Approach}

Given an evacuation network with each edge having a capacity and travel time and sourcedestination capacities, with the general contraflow problem or lane reversals consists of finding a network reconfiguration with ideal directions to each lane subject to the given constraints with reallocated available edge capacity, that maximizes a given objective function. In addition to evacuations, the contraflow technique is also applicable in order to reduce congestion and traffic jams during the day to day rush hours like office time, some events management cases or street demonstrations. Authors from different field of research have reported significant time possibilities and an effective adaption of technique.

\subsection{Earliest Arrival Contraflow Problem}

Arulselvan [1] and Rebennack et al [17] have considered the contraflow approach with respect to the computational complexity. Given a directed graph $\mathrm{G}=(\mathrm{V}, \mathrm{E}$,$) with single source s and single$ sink thaving travel time $\tau_{i j} \in \mathrm{T}$ with $\tau_{i j}=\tau_{j i}$ for $(\mathrm{i}, \mathrm{j}),(\mathrm{j}, \mathrm{i}) \in \mathrm{E}$ and capacity $C_{i j} \in z^{+}$for each $(i, j) \in E$. The MDCF problem require to find the maximum amount of flow that can sent within the given integer time $\mathrm{T}$ units from the source to the sink $\mathrm{t}$ if the direction of the arc can be reversed at time zero. The problem becomes earliest arrival contraflow problem if it sends maximum amount of flow from source to the sink for each $\theta, \theta=0,1,2, \ldots, \mathrm{T}$. The earliest arrival contraflow problem was first introduced in [6].

\subsection{Procedure of Maximum Contraflow Problem}

1. Construct the transformed graph $\tilde{G}=(\mathrm{V}, \widetilde{E})$ where the arc set is defined as $(\mathrm{i}, \mathrm{j}) \in \tilde{E}$ if $(\mathrm{i}, \mathrm{j})$, $(\mathrm{j}, \mathrm{i}) \in E$. The arc capacity function is defined by $\tilde{C}_{i j}=C_{i j}+C_{j i}$ for all $\operatorname{arcs}(\mathrm{i}, \mathrm{j}) \in \tilde{E}$ and the transit time is $\tau_{i j}^{\prime}\left(=\tau_{j i}^{\prime}\right)= \begin{cases}\tau_{i j} & \text { if }(i, j) \in E \\ \tau_{j i} & \text { otherwise. }\end{cases}$

2. Solve the maximum flow problem on graph with $\tilde{G}$ capacity $\tilde{C}$.

3. Perform flow decomposition into path and cycle flows of the maximum flow resulting from step 2. Remove the cycle flows.

4. Arc $(\mathrm{j}, i) \in E$ is reversed iff flow along arc $(\mathrm{i}, \mathrm{j})$ is greater than $C_{i j}$ or if there is a non negative flow along arc $(i, j) \in E$ and resulting flow is the maximum flow with arc reversal for graph $\mathrm{G}=(\mathrm{V}, \mathrm{E})$.

Arulselvan et al. [18] presented strongly polynomial time for the single source and single sink MDCF problem and the multi - terminal static contraflow problem. The time complexities of these algorithm are $\mathrm{O}\left[S_{2}(|\mathrm{Vl},| \mathrm{El})+S_{3}(|\mathrm{Vl},| \mathrm{El})\right]$ and $\mathrm{O}\left[S_{1}(\mathrm{Vl}, \mid \mathrm{El})+S_{2}(|\mathrm{Vl},| \mathrm{El} \mid)\right]$ respectively.

The algorithm for the MDCF Algorithm has been modified [6] for the TTSS graph.

Example 2: Consider a dynamic contraflow as given in the Figure 4(a). In Figure 4(b) the arcs of the network from the source to the sink are reversed towards the outgoing arcs at time zero. 
The new increased capacities $C_{i j}$ are obtained by adding capacities of both arcs; however the travel time is $\tau_{i j}$ remain the same. A complication arises on the intersecting paths. For example, which direction of the $(\mathrm{x}, \mathrm{y})$ or $(\mathrm{y}, \mathrm{x})$ with added capacity gives the efficient flow through the network? Here, we are choosing the $\operatorname{arc}(\mathrm{x}, \mathrm{y})$ randomly.

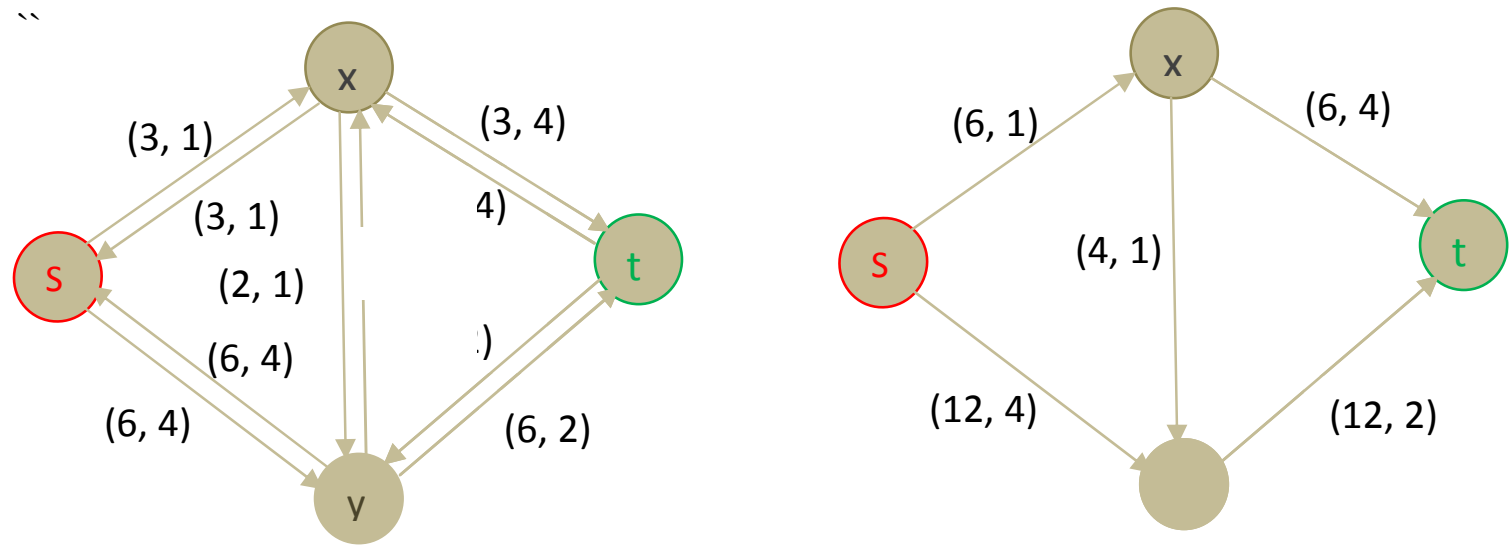

Fig. 4: Evacuation Scenario (a) without Contraflow (b) with Contraflow

For the MDCF problem, we consider maximum flow paths with in time horizon $\mathrm{T}=6$. As shown in figure 4(c), the path $s-x-t$ and $s-y-t$ sufficient for a maximum flow and the arc $(x, y)$ or $(y, x)$ does not affect for the flow value. Figure 4(c) represents the temporally repeated flow obtained by Ford and Fulkerson [8]. The MCDF problem has the same solution according to the algorithm Rebennack et al [18]. But for EACF problem, as we need shortest distance path at all successive time periods, a flow through the arc $(\mathrm{x}, \mathrm{y})$ is essential due to shortest path $\mathrm{s}-\mathrm{x}-\mathrm{y}-\mathrm{t}$ as shown in the figure 4(d). A flow of maximum value 4 is reached to the sink at the earliest time $\mathrm{T}=4$ through the path. But it is not yet guaranteed whether the orientation remains valid for the latter time periods. Note that a solution with this path structure does not represent a temporally repeated flow since the chain flow $\mathrm{s}-\mathrm{x}-\mathrm{y}-\mathrm{t}$ is started only at 0 and 1 but not at 2 , though there would be enough time for that chain flow to reach the sink.

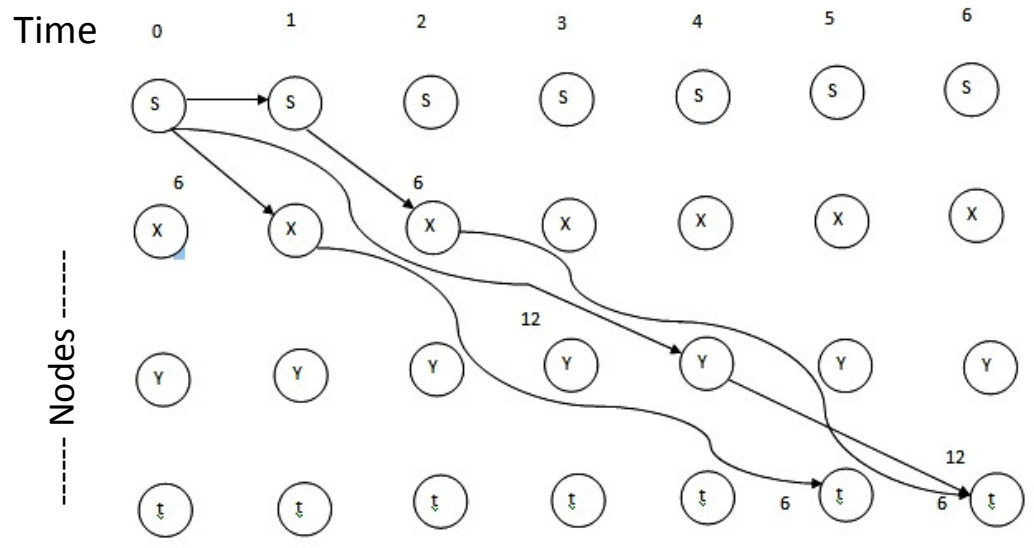

Fig. 4 (c): Maximum Dynamic Contraflow for Fig. 4(b) 


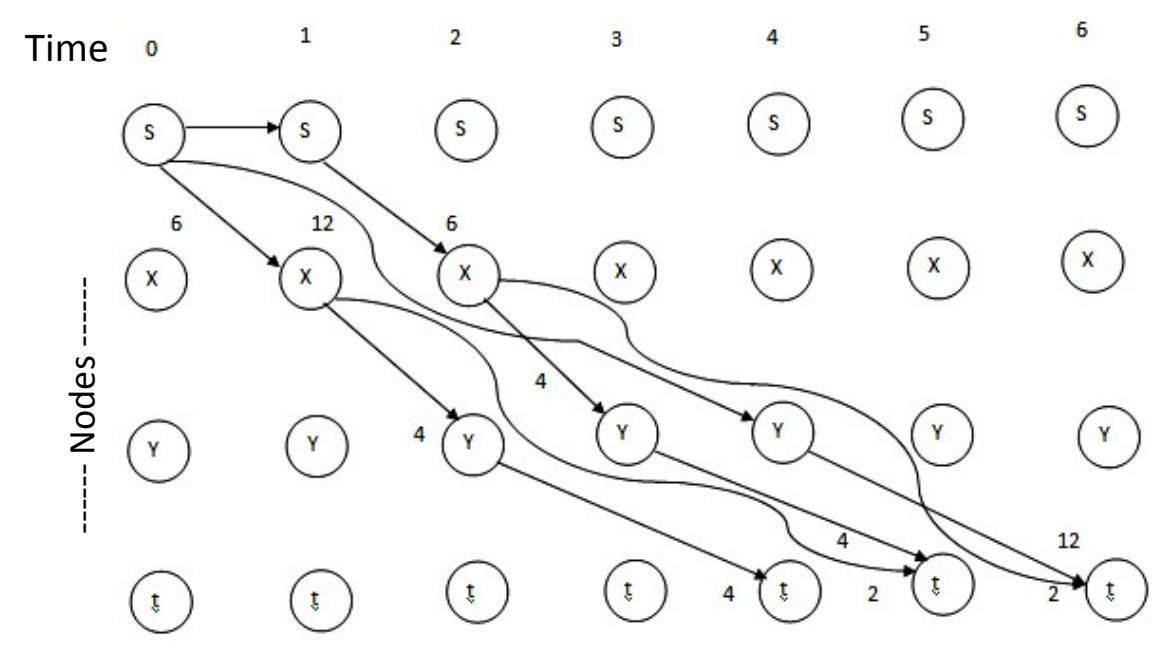

Fig. 4 (d): A Contraflow for Fig. 4(a) with respect to Fig. 4(b).

It is observed from this example that the algorithm of Rebennack et al. [18] is unable to find the MDF solution having the earliest arrival property. Moreover, the network in figure 4 (b) and also with another orientation (y, $x$ ) in figure 4 (a) is not a series parallel graph. Hence the algorithm presented by Rebennack et al [18] could not find the EACF solution unless the graph is series parallel. A complication arises because of the flipping requirements of such intermediate arcs with respect to the time. Dhamala and Pyakurel [6] modify the algorithm in Rebennack et al. [18] for the MDCF problem for TTSP graph that also satisfy the earliest arrival property.

In above we also notice that 12 unit of flow can send from source to the sink in 6 time units before contraflow and after contraflow solution is improved 24 units of flow can send from source to the sink in same time period.

Algorithm 3: [6] Maximum Dynamic Contraflow (MDCF) on TTSP graphs.

1. Construct the transformed graph $\tilde{G}=(\mathrm{V}, \tilde{E})$ where the arc set is defined as $(\mathrm{i}, \mathrm{j}) \in \mathrm{E}$ if $(\mathrm{i}, \mathrm{j}),(\mathrm{j}, \mathrm{i}) \in \mathrm{E}$ the arc capacity function is given by $\tilde{C}_{i j}=C_{i j}+C_{j i}$ for all $\operatorname{arcs}(\mathrm{i} ; \mathrm{j}) \in \tilde{E}$ and the transit time is

$$
\tau_{i j}^{\prime}\left(=\tau_{j i}^{\prime}\right)= \begin{cases}\tau_{i j} & \text { if }(i, j) \in E \\ \tau_{j i} & \text { otherwise }\end{cases}
$$

2. Generate a dynamic temporally repeated flow on transformed series parallel graph $\tilde{G}$ with capacity $\tilde{C}$ and travel time $\tilde{\tau}_{i j}$.

3. Perform flow decomposition into path and cycle flows of the maximum flow resulting from step 2. Remove cycle flows.

4. $\operatorname{Arc}(\mathrm{j}, \mathrm{i}) \in E$ is reversed if and only if the flow along $\operatorname{arc}(\mathrm{i}, \mathrm{j})$ is greater than $C_{i j}$ or if there

is a non negative flow along arc $(\mathrm{i}, \mathrm{j}) \in E$ and the resulting flow is the maximum flow with arc reversal for graph $\mathrm{G}=(\mathrm{V}, \mathrm{E})$. 


\section{Concluding Remarks}

The evacuees can be efficiently sent in a maximum number at every step of time over the time horizon using optimization techniques. The problem can be formulated as a linear programming problem described as the evacuees' flow along the reversed arcs of a dynamic network. There exists a number of efficient algorithms based on the Ford and Fulkerson's approach of sending maximum flow from the source to the sink.

The contraflow approach has been useful to implement during the evacuation to find the efficient routes from the source to the sink. The applicability of the concept has been increased over the past years not only for the evacuation but also to resolve the congestion during the rush hours in the populated city. The general problem with arc reversal cost is computationally hard. It is still open whether there exists a polynomial time algorithm or not. However, there exists a polynomial algorithm on a special class of graph. Moreover, the problem with the case of partial arc reversal is still unsolved.

\section{Acknowledgement}

The research of the first author was supported by University Grant Commission, Sanothimi, Bhaktpur.z

\section{References}

[1] Arulselvan A (2009), Network model for disaster management, Ph D thesis, University of Florida USA.

[2] Baumann N and Skutella M (2006), Earliest arrival flows with multiple sources. Mathematics of operations research, 34(2):499 - 512.

[3] Bein WW, Brucker P and Tamir A (1985), Minimum cost flow algorithm for series parallel networks. Discrete applied Mathematics 10(2): 117- 124.

[4] Brucker P (1982), Network flows in trees and Knapsack problems with nested constraints. Proceeding on $8^{\text {th }}$ Conference on graph theoretic concepts in computer science, Hanser 25-35.

[5] Dhamala TN (2015), A survey models algorithms for discrete evacuation planning network problems. Journal of Industrial and Management Optimization, 11(1): 265 - 289.

[6] Dhamala TN and Pyakurel U (2013), Earliest arrival contraflow problem on series parallel graph. International Journal of Operation Research, 10(1): 1-13.

[7] Dhungana RC, Pyakurel U, Khadka SR and Dhamala TN (2015),Universally maximum contraflow for evacuation planning. International Journal of Operation Research, Nepal, 4(1): 67-78.

[8] Ford LR and Fulkerson DR (1958), Constructing maximal dynamic flows from static network. Operations Research, 6:419- 433.

[9] Ford LR and Fulkerson DR (1962), Flows in network. Princeton University Press, Princeton, New Jersey. 
[10] Gale D (1959), Transient flow in network. Michigan Mathematical Journal, 659-663.

[11] Hoppe B and Tardos E (1994), Polynomial time algorithm for some evacuation problems proceeding of $5^{\text {th }}$ Annual ACM-SIAM symp. on discrete algorithms, 433- 441.

[12] Hoppe B (1995), Efficient dynamic network flow algorithms. PhD thesis, Cornell University.

[13] Kim and Shekhar S (2005), Contraflow network re-configurations for evacuation planning; a summary of result. Proceeding of $13^{\text {th }}$ ACM Symphosium on advances in Geographic Information system GIS, 13: 250-259.

[14] Minieka E (1973), Maximal Lexicographic and Dynamic Network flows. Operations Research, 21: 517-527.

[15] Pyakurel U, Hamacher HW and Dhamala TN (2014), Generalized maximal dynamic Contraflow on lossy network, International Journal of operations Research Nepal, 3(1): 2744.

[16] Pyakurel U and Dhamala TN (2014), Lexicographically contraflow problem for evacuation planning. Second International Conference on Operations Research Nepal, 287-294.

[17] Dhamala TN and Pyakurel U (2015), Models and algorithms on contraflow evacuation planning network problems. International Journal of Operations Research, 12(2): 36 - 46.

[18] Rebennack S, Arulselvan A, Elefteriadou L and Pardalos PM (2010), Complexity analysis of maximum flow problem with arc reversal. Journal of Combinatorial Optimization 29: 200216.

[19] Ruzika S, Sperber H and Steiner M (2011), Earliest arrival flows on series parallel graphs. Networks, 10: 169-173.

[20] Steiner M (2009), A survey on earliest arrival flow and a study of the series parallel cases Diploma thesis, Department of Mathematics, University of Kaiserslautern.

[21] Wilkinson WL (1971), An algorithm for maximal dynamic flows in a network. Operations Research, 19: 1602-1612. 